\title{
A Complete and Efficient Multisphere Scattering Theory for Modeling the Optical Properties of Interplanetary Dust
}

\author{
Yu-Iin Xu and Bo A. S. Gustafson \\ Department of Astronomy, 21/ SSRB, University of Florida, Gainesville, \\ Fl 32611, U.S.A.
}

For a long time, the dominant scattering theory used in radiative transfer and scattering calculations has been Mie theory, which is the complete solution to the problems of light scattering by single, isotropic, and homogeneous spheres. However, cosmic dust collections show that most of the largest sized interplanetary dust particles may be porous, inhomogeneous, and aggregated and may have quite different scattering properties. Arbitrary configurations of aggregated spheres may provide a reasonable first approximation to realistic light-scattering models of interplanetary dust particles. In the last few decades, progress has been made in developing light scattering theory for interacting spheres. The development of the addition theorems for scalar and vector spherical wave functions (Friedman \& Russek, 1954; Stein, 1961; Cruzan, 1962) opened up a new area in the theoretical study of multisphere scattering problems.

The first step toward the solution of the multisphere problem using the method of modal analysis is to solve the boundary conditions for the interactive scattering coefficients of each sphere in a spherical coordinate system with its origin at the center of the sphere. Bruning \& Lo $(1971 \mathrm{a}, \mathrm{b})$ were the first to publish such a solution for two interacting spheres. Fuller \& Kattawar $(1988 \mathrm{a}, \mathrm{b})$ extended Bruning \& Lo's solution pairwise to the case of an arbitrary aggregate of spheres. This solution was also independently obtained by Borghese et al. (1979) using Debye potentials. Recently, Xu (1995) derived the solution from the generalization of the Mie theory and showed that the extended Mie theory directly leads to the linear equations containing the interactive scattering coefficients $\left(a_{m n}^{j}, b_{m n}^{j}\right)$ of the $j$-th sphere in an $L$-sphere aggregate:

$$
\begin{aligned}
& a_{m n}^{j}=a_{n}^{j}\left[p_{m n}^{j}-\sum_{i \neq j}^{(1, L)} \sum_{\nu=1}^{\infty} \sum_{\mu=-\nu}^{\nu}\left(a_{\mu \nu}^{i} A_{m n \mu \nu}^{j j}+b_{\mu \nu}^{i} B_{m n \mu \nu}^{j j}\right)\right], \\
& b_{m n}^{j}=b_{n}^{j}\left[q_{m n}^{j}-\sum_{i \neq j}^{(j, L)} \sum_{\nu=1}^{\infty} \sum_{\mu=-\nu}^{\nu}\left(a_{\mu \nu}^{j} B_{m, n \nu}^{j j}+b_{\mu \nu}^{l} A_{m n \mu \nu}^{i j}\right)\right],
\end{aligned}
$$

where $j=1,2, \ldots, L$; the degree $n$ and order $m$ are integers with $l \leq n<\infty$ and $|m| \leq n_{i} \quad a_{n}^{j}, b_{n}^{j}$ are the well known Mie scattering coefficients of the $j$-th sphere: $p_{m n}^{j}, q_{m n}^{j}$ are the expansion coefficients of the incident electromagnetic field expressed in the $j$-th coordinate system; $A_{m n \mu \nu}^{j}, B_{m n \mu \nu}^{j j}$ are the vector transiational 
addition coefficients introduced by the modal reexpansion from the $l$-th to the $j$-th coordinate system; and $(\mu, \nu)$ have the same definitions as $(m, n)$. The formulation and computation of the huge number of vector translational addition coefficients required by the vector addition theorems is one of the challenging problems in multisphere scattering theory. Direct calculation of the addition coefficients requires the determination of the so-called Gaunt coefficients (Gaunt, 1929) that are defined by the definite integrals of the product of three associated Legendre functions. Recently, $\mathrm{Xu}$ (1996a) devised an efficient algorithm for the fast evaluation of the Gaunt coefficients, which reduces the computing time to as little as $1 \%$ of that required by the $3 \mathrm{jm}$ formulation (Cruzan, 1962). Based on Xu's algorithm, any normalized Gaunt coefficient can be analytically expressed as

$$
\tilde{a}_{q}=\frac{2 p+1}{2} \sum_{i=0}^{q} \frac{(p+q-i+3 / 2)_{q+i-1}}{(q-i) !\left(n_{4}-2 i+1\right)_{2 i}} \sum_{j=0}^{i} \frac{(m-n)_{2 j}(\mu-\nu)_{2 i-2 j}}{j !(i-j) !(-n+1 / 2)_{j}(-\nu+1 / 2)_{i-j}},
$$

where $p=n+\nu-2 q$ and $n_{4}=n+\nu-m-\mu$. The explicit expressions for the vector translational coefficients given by Xu (1996b) are

$$
\begin{aligned}
& A_{m n \mu \nu}^{l j}=\frac{(-1)^{m} i^{\nu+n}(n+2)_{n-1}(\nu+2)_{\nu+1}(n+\nu+m-\mu) !}{4 n(n+\nu+1)_{n+\nu}(n-m) !(\nu+\mu) !} \exp \left[i(\mu-m) \phi_{l j}\right] \\
& \quad \times \sum_{q=0}^{q_{\max }}(-1)^{q}[n(n+1)+\nu(\nu+1)-p(p+1)] \widetilde{a}_{1 q}\left[\begin{array}{c}
z_{p}^{(J)}\left(k d_{l j}\right) \\
j_{p}\left(k d_{l j}\right)
\end{array}\right] P_{p}^{\mu-m}\left(\cos \theta_{l j}\right) \\
& B_{m n \mu \nu}^{l j}=\frac{(-1)^{m} i^{\nu+n+1}(n+2)_{n+1}(\nu+2)_{\nu+1}(n+\nu+m-\mu+1) !}{4 n(n+1)(n+m+1)(n+\nu+2)_{n+\nu+1}(n-m) !(\nu+\mu) !} \\
& \quad \times \exp \left[i(\mu-m) \phi_{l j}\right] \sum_{q=0}^{Q_{\max }}(-1)^{q}\left\{2(n+1)(\nu-\mu) \tilde{a}_{2 q}-[p(p+3)-\nu(\nu+1)\right. \\
& \left.\quad-n(n+3)-2 \mu(n+1)] \tilde{a}_{3 q}\right\}\left[\begin{array}{c}
z_{p+1}^{(J)}\left(k d_{l j}\right) \\
j_{p+1}\left(k d_{l j}\right)
\end{array}\right] P_{p+1}^{\mu-m}\left(\cos \theta_{l j}\right) \quad\left(\begin{array}{c}
r \leq d_{l j} \\
r \geq d_{l j}
\end{array}\right)
\end{aligned}
$$

where $j_{p}$ is the spherical Bessel function of the first kind, and $z_{p}$ is any one of the four spherical Bessel functions; $q_{\max }=\min [n, \nu,(n+\nu-|m-\mu|) / 2]$, $Q_{\max }=\min [n+1, \nu,(n+\nu+1-|m-\mu|) / 2] ;\left(d_{l j}, \theta_{l j}, \phi_{l j}\right)$ are the spherical coordinates of the origin of the $j$-th coordinate system in the $l$-th coordinate system; $\widetilde{a}_{1 q}, \widetilde{a}_{2 q}, \widetilde{a}_{3 q}$ are abbreviated notations for the normalized Gaunt coefficients $\widetilde{a}(-m, n, \mu, \nu, n+v-2 q), \widetilde{a}(-m-1, n+1, \mu+1, \nu, n+v+1-2 q)$, and $\widetilde{a}(-m, n+1, \mu, \nu, n+v+1-2 q)$, respectively.

Once the scattered fields of all spheres in a set are known, the scattering properties of the ensemble or aggregate as a whole can be predicted in terms of the interactive scattering coefficients of the individual spheres. The research conducted on the calculation of the cross sections of clusters of spheres includes that of Borghese $e t$ al. (1979), Géraŕdy \& Ausloos (1982), Mackowski (1991, 1995), Fuller (1995), Xu (1995) and others. The analytical expressions for the four elements of the amplitude scattering matrix have been derived by Xu (1995). A common problem considered in all these works is the construction of the total scattered field from the entire aggregate of spheres. All individual scattered fields need to be reexpanded about an arbitrarily 
chosen common $j_{0}$-th coordinate system. Naturally, the vector addition theorems may be used for the second time and the total scattering coefficients are given by

$a_{m n}=\sum_{l=1}^{L} \sum_{\nu=1}^{\infty} \sum_{\mu=-\nu}^{\nu}\left(A_{m n \mu \nu}^{l_{0}} a_{\mu \nu}^{l}+B_{m n \mu \nu}^{l_{0}} b_{\mu \nu}^{l}\right), b_{m n}=\sum_{l=1}^{L} \sum_{\nu=1}^{\infty} \sum_{\mu=-\nu}^{\nu}\left(B_{m n \mu \nu}^{j_{0}} a_{\mu \nu}^{l}+A_{m n \mu \nu}^{l j_{0}} b_{\mu \nu}^{l}\right)$

where $A_{m n \mu \nu}^{l j_{0}}$ and $B_{m n \mu \nu}^{l j_{0}}$ are essentially the same as $A_{m n \mu \nu}^{l j}$ and $B_{m n \mu \nu}^{l j}$ except that the Hankel functions of the first kind in the expressions for $A_{m n \mu \nu}^{l j}$ and $B_{m n \mu \nu}^{l j}$ are replaced by the Bessel functions of the first kind. However, this reexpansion formulation leads to severe numerical problems. The problem worsens with increasing separations between spheres and it makes the reexpansion method unreliable for use with most models of interplanetary dust. Of practical interest is the scattered field in the far zone of the clusters of spheres. It can be shown that when $r \rightarrow \infty$, the vector translational addition coefficients are orthogonal in both degrees and orders and are given by

$$
A_{m n \mu \nu}^{l j_{0}}=\delta_{m \mu} \delta_{n \nu} \exp \left(-i k \Delta^{l}\right), \quad B_{m n \mu \nu}^{l j_{0}} \equiv 0
$$

from which follows that

$$
a_{m n}=\sum_{l=1}^{L} \exp \left(-i k \Delta^{l}\right) a_{m n}^{l}, \quad b_{m n}=\sum_{l=1}^{L} \exp \left(-i k \Delta^{l}\right) b_{m n}^{l},
$$

where $\Delta^{l}=X^{l} \sin \theta \cos \phi+Y^{l} \sin \theta \sin \phi+Z^{l} \cos \theta$ and $\left(X^{l}, Y^{l}, Z^{l}\right)$ are the Cartesian coordinates of the center of the $l$-th sphere in the $j_{0}$-th coordinate system. This approach to constructing the total far scattered field and the consequent analytical expressions for the scattering properties of aggregates of spheres are free from numerical problems in practical calculations. This solution is valid at all points in space as long as the distance to all spheres is large compared to the size of the ensemble and the wavelength and is thus valid in most practical applications including the study of the zodiacal light and thermal emission.
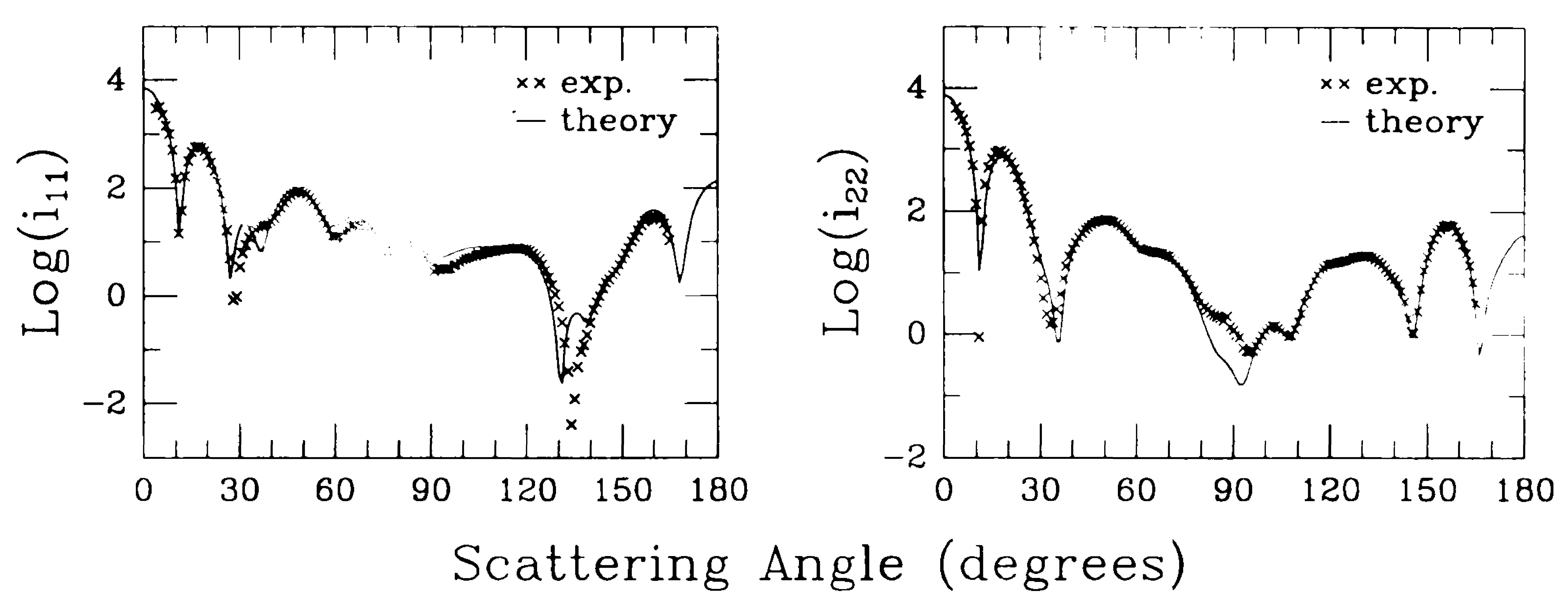

Figure 1. Comparisons of theoretical predictions with laboratory scattering measurements for angular distributions of scattered light by two identical contacting spheres with size parameter of 7.86 and refractive index of $2.511-0.02 i$. The polarization components of scattering intensities $i_{11}$ and $i_{22}$ correspond to the scattered-field components, perpendicular and parallel to the scattering plane, respectively. 
Theoretical predictions from the newly developed multisphere scattering theory for the scattering properties of various configurations of spheres, from bisphere systems to large aggregate of 249 spheres that we tested, are in good agreement with laboratory scattering measurements. An example for a two-sphere system is shown in Fig. 1. The experimental data were obtained through the microwave analog technique using the Laboratory for Astrophysics at the University of Florida (Gustafson, 1996).

In summary, major theoretical and numerical problems in multisphere scattering theory have been purified recently. This cleared the way for the reliable multisphere scattering calculations and a new approach to modeling the interplanetary dust. Thorough experimental scrutiny and systematic numerical tests are still in progress. After its overall validation, we should be able to apply with confidence this multiple scattering theory in the simulations of the optical properties of the interplanetary dust particles. 2482 .

Acknowledgments. This work was supported in part by NASA grant NAGW-

\section{References}

Borghese, F., Denti, P., Toscano, G., \& Sindoni, O. I. 1979, "Electromagnetic scattering by a cluster of spheres," Appl. Opt. 18, 116

Bruning, J. H. \& Lo, Y. T 1971a,b, "Multiple scattering of EM waves by spheres, Part I \& II," IEEE Trans. Ant. Prop. AP-19, 378

Cruzan, O. R. 1962, "Translational addition theorems for spherical vector wave functions," Quart. Appl. Math., 20, 33

Friedman, B. \& Russek, J. 1954, "Addition theorems for spherical waves," Quart. Appl. Math. 12, 13

Fuller, K. A. \& Kattawar, G. W. 1988a,b, "Consummate solution to the problem of classical electromagnetic scattering by ensembles of spheres. I \& II," Opt. Lett. 13, 90-92, 1063

Fuller, K. A. 1995, "Scattering and absorption cross sections of compounded spheres. I. Theory for external aggregation," J. Opt. Soc. Am. A, 11, 3251

Gaunt, J. A. 1929, "On the triplets of helium," Philos. Trans. Roy. Soc. (London) Ser. A228, 151

Gérardy, J. M. \& Ausloos, M. 1982, “Absorption spectrum of clusters of spheres from the general solution of Maxwell's equations. II. Optical properties of aggregated metal spheres," Phys. Rev. B 25, 4204

Gustafson, B. A. S. 1996, "Microwave analog to light scattering measurements; A modern implementation of a proven method to achieve precise control," J. Quant. Spectro. \& Radia. Trans., 55:5, 663

Mackowski, D. W. 1991, "Analysis of radiative scattering for multiple sphere configurations," Proc. R. Soc. London Ser. A, 433, 599

Mackowski, D. W 1995, "Calculation of total cross sections of multiple-sphere clusters," J. Opt. Soc. Am. A, 11, 2851

Stein, S. 1961, "Addition theorems for spherical wave functions," Quart. Appl. Math., 19, 15

$\mathrm{Xu}$, Yu-lin 1995, "Electromagnetic scattering by an aggregate of spheres," Appl. Opt. 34, 4573

Xu, Yu-lin 1996a, "Fast evaluation of the Gaunt coefficients," Math. Comput., in press

$\mathrm{Xu}$, Yu-lin 1996b, "Calculation of the addition coefficients in electromagnetic multisphere-scattering theory," J. Comput. Phys., in press 